\title{
Radial keratotomy: a review of 300 cases
}

\author{
A K Bates, S J Morgan, A D McG Steele
}

\begin{abstract}
Three hundred consecutive cases of radial keratotomy performed between 1985 and 1990 were reviewed. There were no sight threatening complications of surgery and no patient lost one or more lines of corrected Snellen acuity. Overall $78 \cdot 7 \%$ saw $6 / 12$ or better unaided postoperatively and $51.7 \%$ saw $6 / 6$ or better. Refraction showed $61 \cdot 3 \%$ to be within 1 dioptre of emmetropia and $86.7 \%$ were within 2 dioptres. Further analysis demonstrated that results of unaided acuity and proximity to emmetropia were much better for low $(<-2.87 \mathrm{D})$ and moderate $(-3.0$ to -5.87 D) than for high $(>-6.0 \mathrm{D})$ myopes.

(BrF Ophthalmol 1992; 76: 586-589)
\end{abstract}

Currently myopia is being treated by radial keratotomy and by photorefractive keratectomy using the excimer laser. ${ }^{12}$ In order to assess the relative merits of these two techniques the results of radial keratotomy from one surgical team were reviewed over a period of 5 years.

\section{Method}

The notes were reviewed of 300 consecutive radial keratotomy procedures performed between 1985 and 1990 by one surgeon (AD McG S) or directly under his auspices.

Preoperative assessment involved a full ophthalmic examination including mydriasis for fundal examination, confirmation of a stable refraction, and pachymetry. Each patient was thoroughly counselled and the controversial and unpredictable nature of this form of surgery explained. Details of the possible early and late postoperative complications were provided.

All surgery was performed under general anaesthesia. The pupil was constricted preoperatively. The selected size of central clear zone was delineated with a corneal marker fitted with cross wires. Radial incisions were then made with a guarded knife set at $115 \%$ of the measured central corneal thickness. Incisions were made from the edge of the outer clear zone to the periphery, leaving a small margin of uncut cornea at the limbus. Four or eight incisions

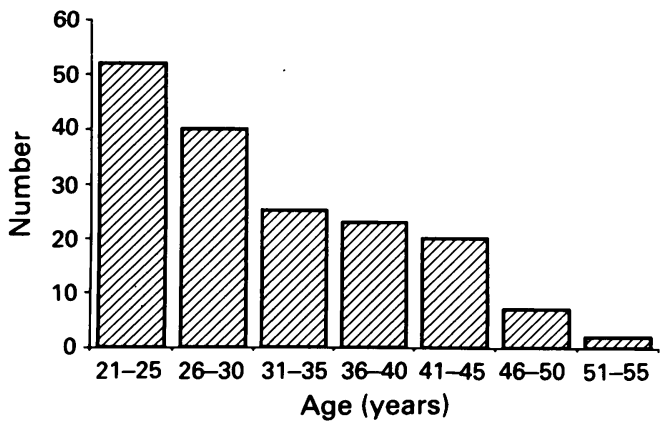

were utilised depending on degree of myopia. Finally the incisions were carefully irrigated with sterile saline, topical antibiotics were instilled, and the eye was padded.

Postoperatively all patients received prednisolone sodium drops $0.3 \%$ and chloramphenicol drops four times daily for 4 weeks. Patients were routinely reviewed at 2 and 6 weeks after surgery and then at $3,6,12$, and 18 months.

\section{Results}

Three hundred procedures were performed on 169 patients of whom 100 were male. All were 21 years of age or older. The age of the patients at surgery is shown in Figure 1 and it may be seen that the majority fall within the 21-30 years age group. Mean time of follow-up was 19 months (range 12-60).

Reasons for wanting surgery are shown in Figure 2. All patients were referred for surgery either directly by their general practitioners $(92 \cdot 8 \%)$ or via other ophthalmologists $(7 \cdot 2 \%)$.

Peroperative complications included 35 cases of single perforation and nine cases in which there were two perforations. All but two were Seidel negative on the morning after surgery, and these were negative by the following morning.

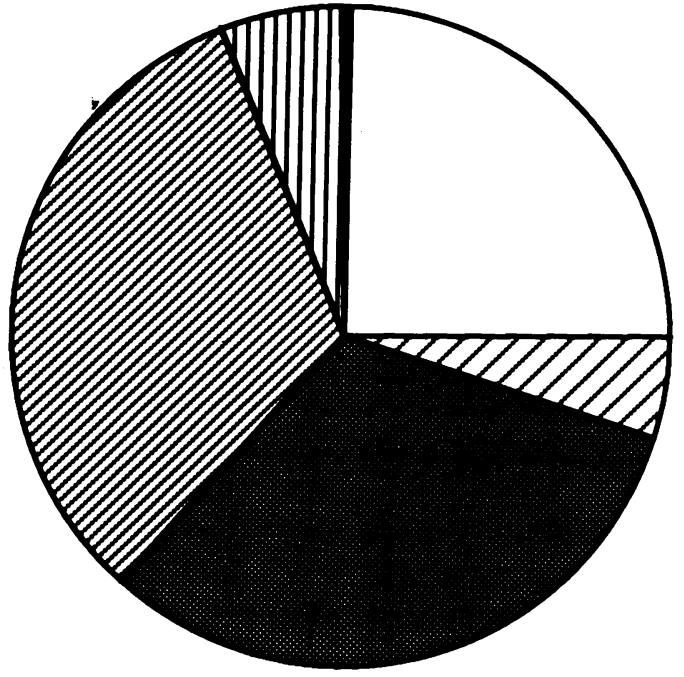
8 April 1992
Accepted for publication

Moorfields Eye Hospital, A K Bates

A Morgan

Correspondence to: 
Figure 3 Unaided visual acuity before and after surgery-all patients.

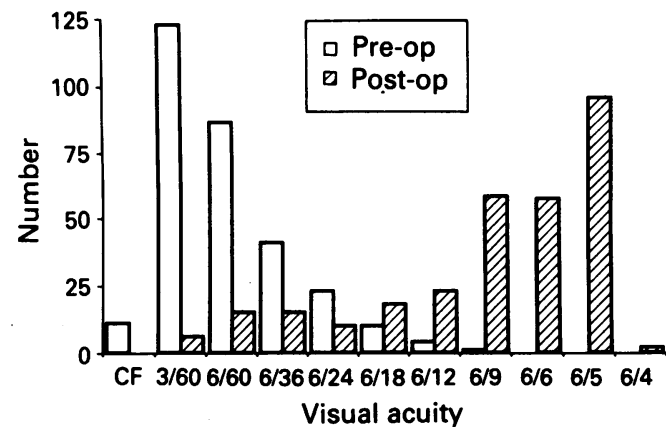

Only one late postoperative complication was recorded; a patient who developed a giant retinal tear 3 months after uneventful surgery which was successfully treated. No other eye in the series lost one line of preoperative corrected Snellen acuity after surgery.

Figure 3 shows unaided Snellen visual acuity before and after surgery for all eyes. The values given here are from the patients' final follow-up visit and this is the case for all results unless otherwise stated. Expressed figuratively $\mathbf{7 8} \cdot \mathbf{7 \%}$ of patients saw $6 / 12$ or better unaided postoperatively and $51 \cdot 7 \%$ saw $6 / 6$ or better.

Spherical equivalent before and after surgery is shown in Figure 4. Expressed figuratively $61.3 \%$ patients were within 1 dioptre of emmetropia postoperatively and $86.7 \%$ were within 2 dioptres.

In order to highlight individual variation in response to surgery Figure 5 shows individual plots of pre- and postoperative refractions. The slope shows the no change line, with all those above the line having a reduction in myopia. This illustrates that the majority of patients with preoperative values of -2 to -6 dioptres achieve results close to emmetropia. Predictability appears to decline with increasing preoperative myopia. To attempt to examine this more closely, eyes were divided into three groups. Low myopes from 0 to -2.87 dioptres, moderate myopes from -3.00 to $-5 \cdot 87$ dioptres, and high myopes of greater than $-6 \cdot 0$ dioptres.

Firstly, postoperative unaided acuity is shown for the three groups in Figure 6. It is clear that predictability of outcome is much poorer in the higher myopes and is best in the low group. This is presented in Table 1 and shows the highest percentage of eyes achieving $6 / 12$ and $6 / 6$ in the low myopia group. The results are progressively poorer for the moderate and high myopia groups. This pattern is repeated with the results of postoperative refraction (Fig 7). The predictability of achieving a refractive result within 1 or

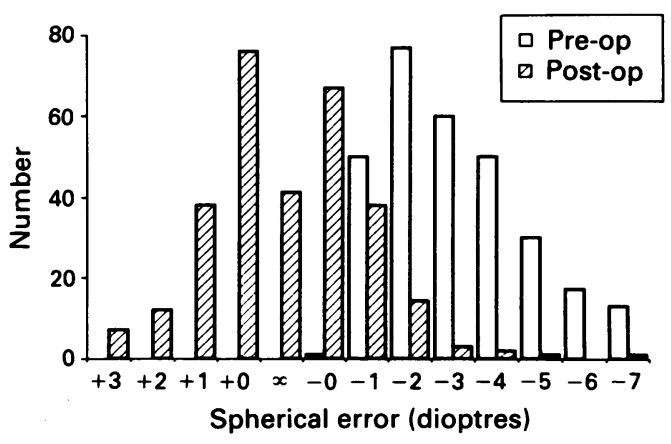

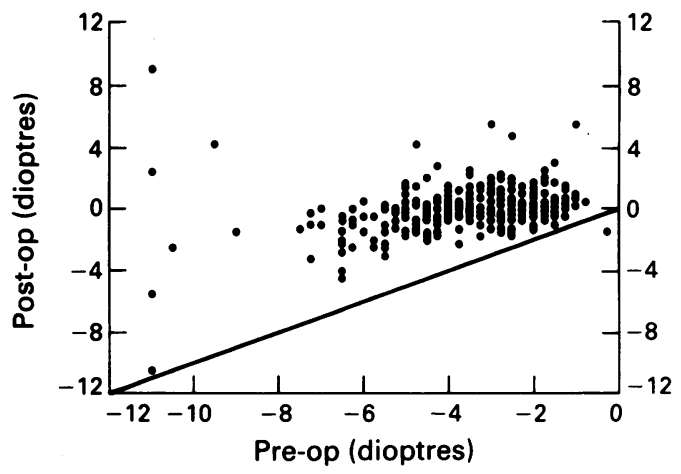

Figure 5 Spherical equivalent before and after surgery individual patients.

2 dioptres of emmetropia declines across these groups from $73 \cdot 8 \%$ to $30 \cdot 0 \%$ and from $93 \cdot 8 \%$ to $56.7 \%$ respectively (Table 2 ).

In order to look at stability of refraction following surgery the spherical equivalent at the refraction nearest to 3 months after surgery was compared with that at the final follow-up visit which was at a mean of 19 months and was always greater than 12 months (Fig 8). This shows that $90 \%$ of eyes change by 1 dioptre or less over this time period and most less than 0.5 dioptre.

The need for a spectacle correction for distance vision, at final follow-up, was assessed. Sixty five per cent were not wearing any correction, 30\% were wearing spectacles (although often only to drive) and $5 \%$ were wearing contact lenses.

Recorded postoperative symptoms were also analysed. The most common complaint at discharge was of diurnal variation in vision in 57 eyes. The other principal complaint was of glare or 'starburst' effect in 13 eyes which was principally experienced when driving at night. However in none of these patients were symptoms sufficiently bothersome to prevent them having surgery on their second eye.

\section{Discussion}

Radial keratotomy has been performed at Moorfields since 1981 and more than 600 procedures have been performed by one surgical team. In this series we have reviewed the results from 300 consecutive procedures performed between 1985 and 1990.

The complication rate was low with no sight threatening complications and no patient lost a line of preoperative corrected Snellen acuity as a result of surgery.

A large percentage of patients underwent

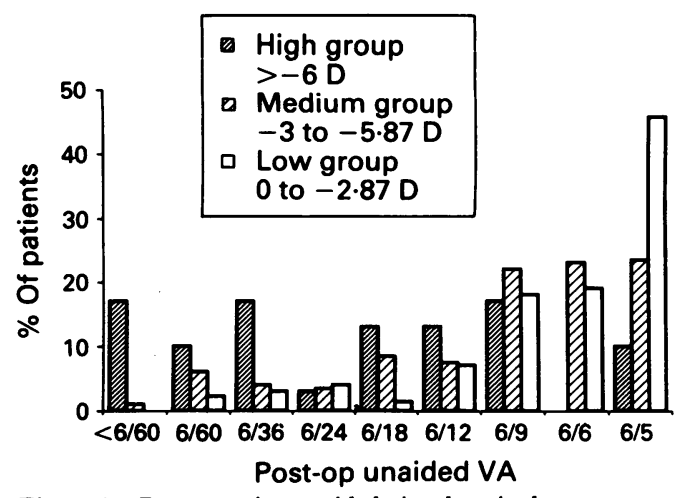

Figure 6 Postoperative unaided visual acuity by groups. 
Table 1 Postoperative unaided visual acuity by groups

\begin{tabular}{llll}
\hline & Low & Mod & High \\
\hline \% within 1 D of emmetropia & 73.8 & 56.5 & 30.0 \\
\% within 2 D of emmetropia & 93.8 & 86.5 & 56.7 \\
\hline
\end{tabular}

surgery to meet the unaided visual requirements of a variety of occupations such as the armed forces, fire service, or prison service. Another large group were having difficulties with their contact lenses and most of the remainder wished to have surgery for cosmetic reasons (Fig 2).

In this series $80 \%$ saw $6 / 12$ or better unaided postoperatively and $52 \%$ saw $6 / 6$ or better (Fig 3 ). This compares with other series such as the 3 year results from the PERK trial where $76 \%$ achieved $6 / 12$ and 52\%, 6/6. ${ }^{3}$ Postoperatively $61 \%$ were within 1 dioptre of emmetropia and $86.7 \%$ were within 2 dioptres of emmetropia. This compares with the 3 year figures from the PERK series of $58 \%$ and $81 \%$. $^{3}$

Thus the group as a whole achieved good results both in terms of unaided visual acuity and spherical equivalent. However, as with all refractive surgery there is variation in the effect, and the results of individual patients rather than the group as a whole (Fig 5) show that this particularly affects those patients with high levels of myopia, although there was one low myope who did not respond to surgery. It is important to note that it is not simply a question of less reduction in myopia at the higher levels of attempted correction, but much greater unpredictability shown by those eyes from this group which became significantly hypermetropic. This finding has also been described in other studies. $^{\text {t5 }}$

In order to attempt to confirm this trend of declining predictability of surgery with increasing preoperative myopia, patients were divided into three groups on the basis of degree of myopia. Results were examined from low, moderate, and high myopes with 0 to $-2 \cdot 87$, -3.0 to $-5 \cdot 87$, and -6.00 dioptres or more respectively (Figs 6 and 7 and Tables 1 and 2). Both the unaided visual acuities and refractive results clearly demonstrate that the most accurate correction occurred in the low myopia group with $89 \%$ achieving $6 / 12$ vision or better and $94 \%$ within 2 dioptres of emmetropia. The results for the group of moderate myopes may also be considered satisfactory since $76 \%$ achieved $6 / 12$ or better and $86 \%$ were within 2 dioptres of
Table 2 Postoperative spherical equivalent by groups

\begin{tabular}{llll}
\hline & Low & Mod & High \\
\hline \% achieving 6/12 or better & $89 \cdot 2$ & $76 \cdot 5$ & $40 \cdot 0$ \\
\% achieving 6/6 or better & $64 \cdot 6$ & $46 \cdot 5$ & $10 \cdot 0$ \\
\hline
\end{tabular}

emmetropia. However the results from the high myopia group were disappointing with only $40 \%$ achieving $6 / 12$ or better and $57 \%$ within 2 dioptres of emmetropia. Other series have reported similar findings. ${ }^{3+}$

If myopia remains significantly undercorrected after surgery the question arises of when further surgery should be undertaken. Recent studies have drawn attention to the possibility of a late hypermetropic shift in some patients after radial keratotomy. ${ }^{67}$ Our results showed only small changes in refraction between 3 months after surgery and final follow up (Fig 8), at a mean of 19 months (range 12-60 months). Although this does not exclude a very late diminution in the effect of surgery it would seem reasonable to embark upon further surgery for undercorrection of myopia at 3 months after the initial procedure.

For patients, perhaps one of the most important considerations in the decision as to whether to undergo surgical treatment of myopia, is the likelihood of being able to perform most daily tasks without optical correction. In this series two thirds wore no distance correction at final follow-up, and many others used spectacles only when driving.

The retrospective nature of this study does not allow a comprehensive account of patients' symptoms. Minor visual complaints appeared to be common, especially of diurnal variation in unaided vision and glare when driving at night, but none sufficiently severe to dissuade patients from surgery on their other eye. The only patients who decided not to proceed with surgery on the second eye did so because of a poor refractive result in the first eye.

Radial keratotomy offers fairly accurate correction of myopia of between 2 and 6 dioptres. Outside this range results become unpredictable. Side effects such as diurnal variation in refraction and glare were common but generally well tolerated, perhaps because patients had been carefully counselled prior to surgery about the likelihood of such effects.

Severe complications were rare in this series, but those reported elsewhere include endophthalmitis, ${ }^{8}$ delayed microbial keratitis, ${ }^{10}$ and increased susceptibility to traumatic rupture of the globe. ${ }^{11}$
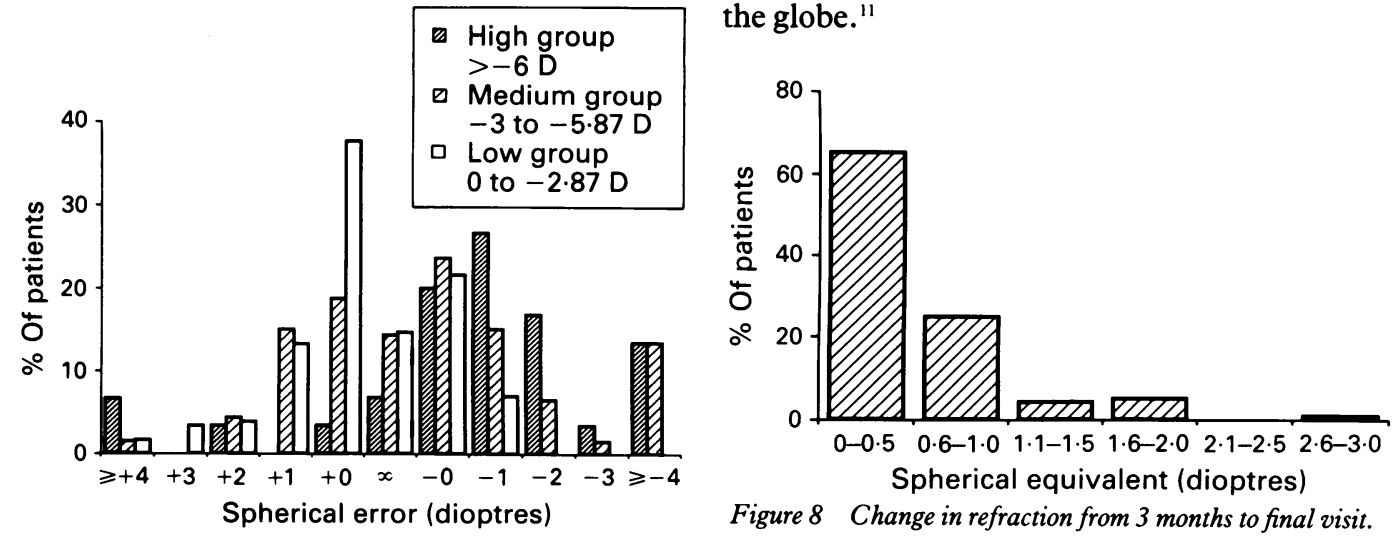
It is hoped that excimer laser photorefractive keratectomy will improve the predictability of correction of myopia and reduce concerns regarding potentially serious complications. The results from radial keratotomy will at least allow an objective comparison for this new technique.

1 Seiler T, Wollensack J. Myopic photorefractive keratectomy with the excimer laser. One year follow up. Ophthalmology 1991; 98: 1156-63.

2 McDonald MB, Liu JC, Byrd TJ, et al. Central photorefractive keratectomy for myopia. Partially sighted and normal eyes. Ophthalmology 1991; 98: 1327-37.

3 Waring GO, Lynn MJ, Culbertson W, et al. Three year results of the prospective evaluation of radial keratotomy (PERK) study. Ophthalmology 1987; 94: 1339-54.

4 Deitz MR, Sanders DR, Marks RJ. Radial keratotomy: an overview of the Kansas City study. Ophthalmology 1983; 91: 467-78.
5 Waring GO, Lynn MJ, Gelender $\mathrm{H}$, et al. Results of the prospective evaluation of radial keratotomy (PERK) study one year after surgery. Ophthalmology 1985; 92: study one year after surgery. Ophthalmology 1985; 92:

6 Deitz MR, Sanders DR. Progressive hyperopia with long term follow up of radial keratotomy. Arch Ophthalmol 1985; 103: $782-4$.

7 Waring GO, Lynn MJ, Strahlman ER, et al. Stability of refraction during four years after radial keratotomy in the prospective evaluation of radial keratotomy study. Am $\mathcal{F}$ Ophthalmol 1991; 111: 133-44.

8.Gelender H, Flynn HW, Mandelbaum SH. Bacterial endophthalmitis resulting from radial keratotomy. $A m \mathcal{F}$ Ophthalmol 1982; 93: 323-5.

9 Mandelbaum S, Waring GO, Forster RK, et al. Late development of ulcerative keratitis in radial keratotomy scars. Arch Ophthalmol 1986; 104: 1156-8.

10 Shivitz IA, Arrowsmith PN. Delayed keratitis after radial keratotomy. Arch Ophthalmol 1986; 104: 1153-5.

11 Binder PS, Waring GO, Arrowsmith PN, et al. Histopathology of traumatic corneal rupture after radial keratotomy. Arch Ophthalmol 1988; 106: 1584-90. 